\title{
An Optimization Problem and Method for Bin Location in Urban Areas
}

\author{
Weixuan Shi ${ }^{1}$, Qi Jiang ${ }^{1}$, Meng Zhang ${ }^{1, *}$ and Qunli Yuchi ${ }^{2}$ \\ ${ }^{1}$ School of Management, Xi'an Jiaotong University, $710049 \mathrm{Xi}$ 'an, China \\ ${ }^{2}$ School of Economics and Management, Xi'an University of Technology, 710054 Xi'an, China \\ *Corresponding author. Email: zm890629@sina.com
}

\begin{abstract}
Bin location in urban areas is a significant research problem. Prior research has considered the average distance that citizens reach bins and it turns out that a citizen may walk a very long distance to dispose the waste. In this paper, we intend to optimize the locating of bins in urban areas. By minimizing the maximum distance for citizens to bins, we find out the optimal location of a bin on a road. Then, a bi-objective model is developed to optimize the locations of bins in a road network, in which one objective is to minimize the maximum distance for citizens to bins, while the other minimizes the installation costs of bins. The solution method and a case analysis based on a real road network in Xi'an are given. The results verify the effectiveness of the model and method.
\end{abstract}

Keywords: Bin location, Urban areas, Maximum distance, Bi-objective.

\section{INTRODUCTION}

With the advance of green development, more and more countries lay stress on processing, recycling and disposing urban waste. For example, China strives to build a waste-free society to develop an ecological civilization and build a Beautiful China. The effective treatment of urban waste needs to make sure that the rubbish would be thrown into bins. However, the actual problem is that the rubbish would be littered with a high possibility if a resident is far from the bin. Therefore, reasonable installation of bins is of great importance to abate pollution, improve environment and promote green development.

A number of studies have focused on modeling the locations of landfills, for example, Yadav et al. [1], Eiselt and Marianov [2], Habibi et al. [3]. There is very little research investigating the installation of rubbish bins. Adeleke and Ali [4] developed a Lagrange heuristic to optimize the location of waste collection sites by considering different types of waste with the aim of minimizing the total number of waste collection sites and bins. Erfani et al. [5] have studied the storage bins locations and vehicle routing systems with the purpose of increasing the efficiencies of waste collection and reducing the collection costs. Blazquez and Paredes-Belmar [6] considered the minimum costs and presented mixed integer linear programming models to decide the number, location and type of bins. As noted in the prior literature, to minimize the costs has gained prominence. It is worth noting that Rossit et al. [7] have proposed an exact multi-objective approach to deal with the bin locations by taking optimization criteria into consideration, including the accessibility to the system, the waste bins installment costs and the waste removal frequency of bins. Having considered the accessibility to the system which can be affected by the distance that a resident reaches bins, the aforementioned study revealed the fact that some residents are unwilling to walk a long distance so that they may dump the waste in unsuitable places.

This paper probes into the bin location problem, focusing on the maximum distance for citizens to bins. By minimizing the maximum distance for citizens to bins, we find out the optimal location of a bin on a road. Then, a model is formulated as a bi-objective optimization problem to optimize the locations of bins in a road network, in which one objective is to minimize the maximum distance for citizens to bins, while the other minimizes the installation costs of bins. The solution method and a case analysis based on a real road network in Xi'an are given, thus providing theoretical basis for the optimization of bin locations in urban areas. 


\section{THE OPTIMIZATION OF BIN LOCATION ON A ROAD}

In order to simplify the extremely complicated problem for optimization of bin locations, this study first discusses the optimization of bin location on a road.

\subsection{Problem Description}

Give a road $e_{i j}=\left(v_{i}, v_{j}\right) . v_{i}$ and $v_{j}$ are the two nodes of $e_{i j}$, while $l_{i j}$ is the length of $e_{i j}$. Citizens who want to throw away rubbish may be at any site on $e_{i j}$, as shown in Figure 1. It is necessary to find out the installation location of a bin on $e_{i j}$ so that the maximum distance for the furthest citizen to the bin is the shortest.

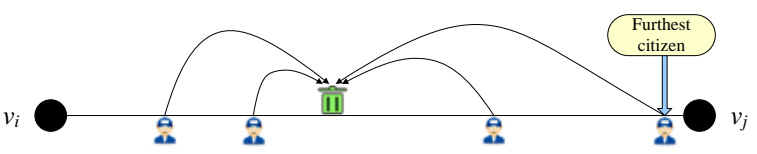

Figure 1 Optimization of bin location on a road.

\subsection{Discussion and Mathematical Formulation}

Let $d_{i j}$ be the distance between the bin and node $v_{i}$ on $e_{i j}$, so $d_{i j}$ is the location of the bin. $l_{i j}^{\prime}$ is the distance between any citizen and node $v_{i}$ on $e_{i j}$. As for the location of citizens who want to throw away rubbish and the bin on $e_{i j}$, there are two cases.

Case 1. $l_{i j}^{\prime} \leq d_{i j}$, then the citizen is closer to node $v_{i}$, as shown in Figure 2(a). The distance between the citizen and the bin is $D_{1}=d_{i j}-l_{i j}^{\prime}$. Obviously, the furthest citizen to the bin should be situated at node $v_{i}$, thus $l_{i j}^{\prime}=0$. The maximum distance for citizens to the bin is $\max D_{1}=d_{i j}$.

Case 2. $d_{i j}<l_{i j}^{\prime}$, then the citizen is closer to node $v_{j}$, as shown in Figure 2(b). The distance between the citizen and the bin is $D_{2}=l_{i j}^{\prime}-d_{i j}$. It is obvious that the furthest citizen to the bin should be situated at node $v_{j}$, thus $l_{i j}^{\prime}=l_{i j}$. The maximum distance for citizens to the bin is $\max D_{2}=l_{i j}-d_{i j}$.

According to the above cases, the optimal location of a bin on road $e_{i j}$ should be $\max D_{1}=\max D_{2}$, to put it another way, $d_{i j}=l_{i j}-d_{i j}, d_{i j}=l_{i j} / 2$. Therefore, the following property is given.
Property 1. If only one bin is installed on a road, the optimal location of the bin should be at the site which is half the length of the road.

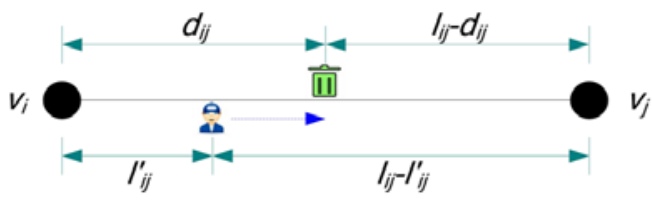

(a) Case 1.

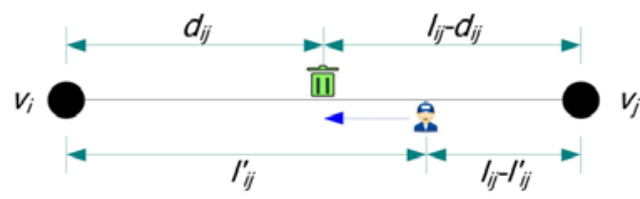

(b) Case 2

Figure 2 The location of a citizen and a bin.

\section{THE OPTIMIZATION OF BIN LOCATIONS IN A ROAD NETWORK}

Having discussed the optimization of a bin on a road, we investigate the optimization of bin locations in a road network in this section.

\subsection{Problem Description and Mathematical Formulation}

\subsubsection{Problem Description}

Give a road network $G(V, E)$, where $V=\left\{v_{1}, v_{2}, \mathrm{~L}, v_{n}\right\} \quad$ is the set of nodes and $E=\left\{e_{i j}\right\}(|E|=m)$ is the set of edges/roads. $l_{i j}$ is the length of $e_{i j}$. Citizens who want to throw away rubbish may be at any site in $G$. The decision maker needs to install bins in $G$, and the unit cost for each bin is $C$. It is necessary to find out the installation locations of bins in $G$ to minimize the maximum distance for the furthest citizen to bins and the installation costs.

For the sake of discussion, we give the following assumptions:

(1) There are at least two bins in a road network.

(2) Citizens have already known the typology of the network and the locations of bins.

(3) If the citizens on one road who want to throw away the rubbish and there is a bin on the road, they prefer to walk to this bin. 
(4) There is at most one bin installed on each road and the location of the bin should be at the site that is one half the length of the road.

\subsubsection{Discussion and Mathematical Formulation}

For citizens on $e_{i j}$, if there is a bin on $e_{i j}$, the maximum distance for citizens to the bin is $l_{i j} / 2$. Otherwise, citizens on $e_{i j}$ need to walk to a bin located on the other roads. The path of citizens should be considered at the first place. Suppose that citizens on $e_{i j}$ need to walk to $e_{g h}$, there are four kinds of routes:

$$
\begin{aligned}
& \text { (1) citizen } \rightarrow v_{i} \rightarrow v_{g} \rightarrow \text { bin, } \\
& \text { (2) citizen } \rightarrow v_{i} \rightarrow v_{h} \rightarrow \text { bin, } \\
& \text { (3) citizen } \rightarrow v_{j} \rightarrow v_{g} \rightarrow \text { bin , } \\
& \text { (4) citizen } \rightarrow v_{j} \rightarrow v_{h} \rightarrow \text { bin }
\end{aligned}
$$

For the convenience of expression, let $S P_{i j g h}=\min \left\{s p_{i g}, s p_{i h}, s p_{j g}, s p_{j h}\right\} \quad, \quad$ where $s p_{i g}, s p_{i h}, s p_{j g}, s p_{j h}$ are the lengths of shortest paths between two nodes.

Citizens on $e_{i j}$ would always have alternative bins located on the other two roads. Suppose that the alternative bins are respectively located on $e_{g h}$ and $e_{a b}$. The worst situation is that citizens on $e_{i j}$ access to the two bins with equal distance. Under this circumstances, the distance between the two bins is $l_{g h} / 2+S P_{i j g h}+l_{i j}+S P_{i j a b}+l_{a b} / 2$, and the length that the citizen need to walk is $\left(l_{g h} / 2+S P_{i j g h}+l_{i j}+S P_{i j a b}+l_{a b} / 2\right) / 2$. The exception that the center of the path between two bins is not on $e_{i j}$ will not be considered in this study yet.

Based on the above analysis, it is still very difficult to solve the optimization problem. Therefore, this study transforms the original problem into the following one.

The edges in the original network can be transformed into nodes, creating a new network $G^{\prime}$. There are $m$ edges in $G$, thus letting the nodes in $G^{\prime}$ being $w_{1}, w_{2}, \mathrm{~L}, w_{m}$. The transformed node $w_{I}$ refers to the site that is half of the length of the corresponding edge in $G$. Figure 3 is an example of transforming the network. $d_{I J}$ is the distance between $w_{I}$ and $w_{J}$. Use $x_{I}$, a 0-1 decision variable, to denote whether to install a bin at $w_{I}$ or not. The mathematic model can be formulated as follows.
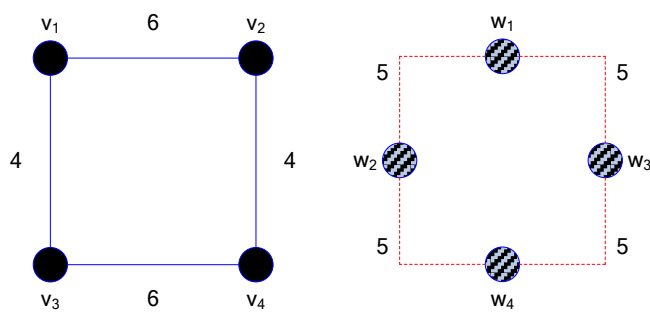

(a) The original network. (b) The transformed network. Figure 3 An example of transforming the network.

$$
\begin{aligned}
& \min f_{1}=\max _{I}\left\{\min \left\{l_{I} / 2+\left(1-x_{I}\right) M, D_{I} / 2+x_{I} M\right\}\right\} \\
\min f_{2}= & \sum_{I=1}^{m} c X_{I}
\end{aligned}
$$

s.t.

$$
\begin{gathered}
D_{I}=\min _{H, J}\left\{d_{H I}+\left(1-x_{H}\right) M+d_{I J}+\left(1-x_{J}\right) M\right\} \\
(H, I, J=1,2, \mathrm{~L}, m ; H \neq I \neq J)
\end{gathered}
$$

$2 \leq \sum_{I=1}^{m} x_{I} \leq m$

$X_{I} \in\{0,1\}$

Objective function (1) means to minimize the maximum distance for citizens to bins, and $M$ is a positive number which is large enough. Objective function (2) refers to minimizing the installation costs of bins. Constraint (3) is the length of the path between two bins. Constraint (4) is to limit the number of installed bins. Compared with the original problem, this model is a much simpler bi-objective optimization model. However, during the process of transformation, to compute $d_{I J}$ requires the use of Dijktra's algorithm $n$ times, and the time complexity is $O\left(n^{3}\right)$.

\subsection{Solution Method and Case Analysis}

\subsubsection{Solution Method}

In this study, we propose a bi-objective optimization model. $\varepsilon$ constraint method is usually used to solve the multi-objective optimization problems, which has been improved to be able to obtain the entire Pareto front, for example, Wang et al. [8], Zhang et al. [9]. The $\varepsilon$ constraint method proposed by Berube et al. [10] is a good case in point. Therefore, this study applies $\varepsilon$ constraint method to compute the results. The steps of the entire solution method are shown below:

(1) Use Dijktra's algorithm to compute all $S P_{i j g h}$ and calculate all $d_{I J}$, then transform $G$ into $G^{\prime}$. 
(2) Combine $\varepsilon$ constraint method and CPLEX to solve the proposed bi-objective model, and obtain the Pareto front.

(3) Choose the preferred solution from the Pareto front and output the plan of bin installation.

\subsubsection{Case Analysis}

The partial real road network in Xi'an is selected as a case study in this research. As is shown in Figure 4, the number on the edge represents the length of the road (unit: hectometer). Let the installation costs of each bin being $c=1$. The mathematical model and solution method presented in this paper are applied to obtain the Pareto front with five points (non-dominated solutions), as shown in Figure 5(a). Choose one of the solutions and present the corresponding plan of bins installation, as Figure 5(b) shows, the dotted line indicates the maximum distance for citizens to bins. Therefore, the model developed and the solutions used in this paper can be applied to effective installation of rubbish bins.

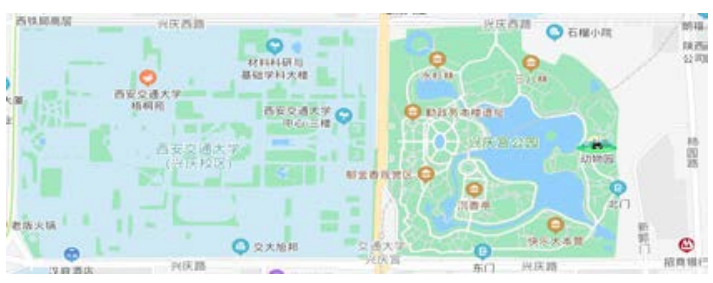

(a) Partial real road network in Xi'an.

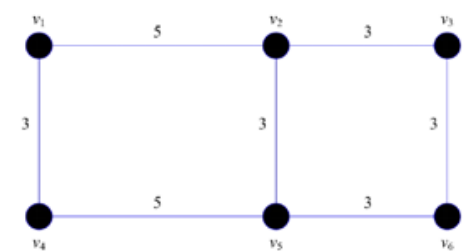

(b) Abstract diagram of original network.

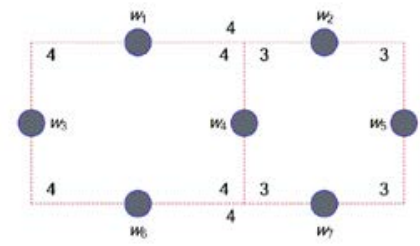

(c) Abstract diagram of transformed network.

Figure 4 Partial real road network in Xi'an and its abstract diagram.

\section{CONCLUSION}

The optimization of bin locations in urban areas is an important research problem. This paper focuses on the maximum distance for citizens to bins to optimize the bin locations in urban areas. First, the optimization of bin location on a road is studied with the aim of minimizing the maximum distance for citizens to bins to find out the optimal location for bin installation. Then, a model is developed to optimize the bin locations in a road network for the purpose of minimizing the maximum distance for citizens to bins and minimizing the installation costs of bins. The model is solved for the data of a real road network in Xi'an. Computational results verify the effectiveness of the model and method.

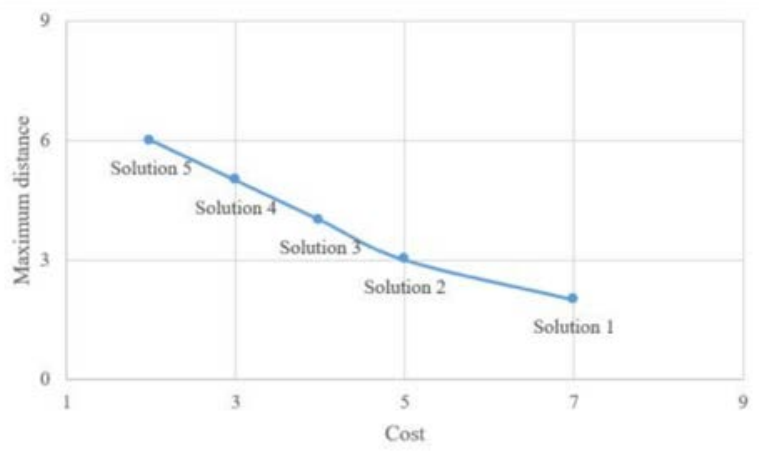

(a) Pareto front.

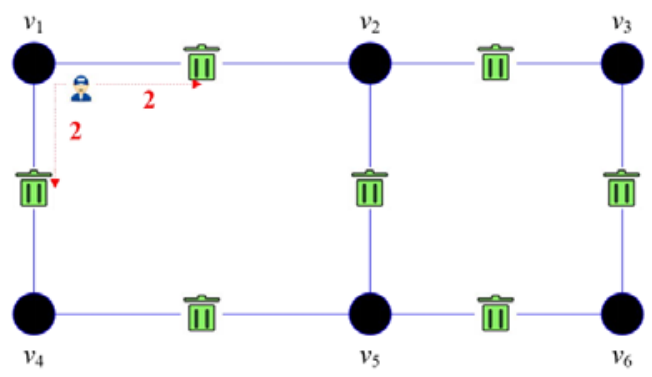

(b) Plan of solution 1.

Figure 5 Pareto front and Solution 1.

\section{ACKNOWLEDGMENTS}

This research is supported by the China Postdoctoral Science Foundation under Grant 2020M683518; the Fundamental Research Funds for the Central Universities under Grant SK2020031; the Special Project of Humanities and Social Sciences of Shaanxi Provincial Department of Education under Grant 20JK0243.

\section{REFERENCES}

[1] Yadav, V., Bhurjee, A.K., Karmakar, S., Dikshit, A.K.: A Facility Location Model for Municipal Solid Waste Management System under Uncertain Environment. Sci. Total Environ. 2017, 603, pp. 760-771.

[2] Eiselt, H.A., Marianov, V.: A Bi-objective Model for the Location of Landfills for Municipal Solid Waste. Eur. J. Oper. Res. 2014, 235, pp. 187-194. 
[3] Habibi, F., Asadi, E., Sadjadi, S.J., Barzinpour, F.: A Multi-objective Robust Optimization Model for Site-selection and Capacity Allocation of Municipal Solid Waste Facilities: A Case Study in Tehran. J. Clean. Prod. 2017, 166, pp. 816-834.

[4] Adeleke, O.J., Ali, M.M.: An Efficient Model for Locating Solid Waste Collection Sites in Urban Residential Areas. Int. J. Prod. Res. 2021, 59, pp. 798-812.

[5] Erfani, S.M.H., Danesh, S., Karrabi, S.M., Shad, R.: A Novel Approach to Find and Optimize Bin Locations and Collection Routes Using a Geographic Information System. Waste Manag. Res. 2017, 35, pp. 776-785.

[6] Blazquez, C., Paredes-Belmar, G.: Network Design of a Household Waste Collection System: A Case Study of the Commune of Renca in Santiago, Chile’. Waste Manag. Res. 2020, 116, pp. 179-189.

[7] Rossit, D.G., Toutouh, J., Nesmachnow, S.: Exact and Heuristic Approaches for Multi-objective Garbage Accumulation Points Location in Real Scenarios. Waste Manag. 2020, 105, pp. 467-481.
[8] Wang, N., Zhang, M., Che, A., Jiang, B.: Biobjective Vehicle Routing for Hazardous Materials Transportation with No Vehicles Travelling in Echelon. IEEE T. Intell. Transp. 2018, 19, pp. 1867-1879.

[9] Zhang, M., Wang, N., He, Z., Yang, Z., Guan, Y.: Bi-objective Vehicle Routing for Hazardous Materials Transportation with Actual Load Dependent Risks and Considering the Risk of Each Vehicle. IEEE T. Eng. Manage. 2019, 66, pp. 429442.

[10] Berube, J., Gendreau, M., Potvin, J.: An Exact $\varepsilon-$ constraint Method for Bi-objective Combinatorial Optimization Problems: Application to the Traveling Salesman Problem with Profits. Eur. J. Oper. Res. 2009, 194, pp. 39-50. 